\title{
Coenzyme Q10 Regulates Antioxidative Stress and Autophagy in Acute Myocardial Ischemia-Reperfusion Injury
}

\author{
Shao Liang, ${ }^{1}$ Zhang Ping, ${ }^{2}$ and Jin $\mathrm{Ge}^{1}$ \\ ${ }^{1}$ Department of Cardiology, The Second Affiliated Hospital and Yuying Children's Hospital of Wenzhou Medical University, \\ Wenzhou, Zhejiang Province, China \\ ${ }^{2}$ Department of Geriatrics \& Neurology, The Second Affiliated Hospital and Yuying Children's Hospital of Wenzhou \\ Medical University, Wenzhou, Zhejiang Province, China
}

Correspondence should be addressed to Zhang Ping; zhangkiki520@126.com and Jin Ge; gemason@126.com

Received 24 April 2017; Revised 19 June 2017; Accepted 26 July 2017; Published 19 November 2017

Academic Editor: Francisco J. Romero

Copyright (c) 2017 Shao Liang et al. This is an open access article distributed under the Creative Commons Attribution License, which permits unrestricted use, distribution, and reproduction in any medium, provided the original work is properly cited.

Background. Oxidative stress and autophagy both play key roles in continuous cardiomyocyte death and cardiac dysfunction after reperfusion therapy for acute myocardial ischemia-reperfusion injury. Coenzyme Q10 (CQ10), which is a fat-soluble quinone antioxidant, is involved in the pathophysiological processes of neurodegenerative diseases, cancer, diabetes, heart failure, and other diseases. Our objective was to determine if, and by what mechanism, CQ10 can ameliorate acute myocardial ischemiareperfusion injury and improve heart function. Methods and Results. Fat-soluble CQ10 in soybean oil solvent was preconditioned in rats with acute myocardial ischemia-reperfusion injury by intraperitoneal injection. Oxidant and antioxidant levels were compared between the preconditioned and control groups. Autophagy was measured by Western blotting analysis of autophagy proteins. Proapoptotic proteins and immunofluorescence were used to assess cell apoptosis. Infarct size was determined by triphenyl tetrazolium chloride (TTC) staining and Evans blue staining and visualized myocardial pathology by tissue staining. Finally, we assessed cardiac function by electrocardiography (ECG) and hemodynamics. Conclusions. This study reveals that CQ10 preconditioning regulates antioxidant levels and the oxidant balance, enhances autophagy, reduces myocardial apoptosis and death, and improves cardiac function in rats with acute ischemia-reperfusion injury. These results imply that CQ10 protects against acute myocardial ischemia-reperfusion injury via the antioxidative stress and autophagy pathways.

\section{Introduction}

Percutaneous coronary intervention is a principal therapeutic approach for the treatment of acute myocardial infarction, which has threatened the life and health of millions of people in recent years [1]. However, some patients still suffer from continuous myocardial cell death, further deterioration of cardiac function, and lower long-term survival rates [2]. The potential mechanisms of ischemia-reperfusion injury are complex and may include distal and/or collateral microcirculation, acute oxygen stress injury, mitochondrial damage, and inflammation [3-6].

Oxidative stress is particularly important in ischemiareperfusion injury through cellular homeostasis, mitosis, cell differentiation, and intracellular signal transduction, which result in cell membrane rupture, swelling, or death [7]. Disruption of the balance between oxidants and antioxidants is serious in acute ischemia-reperfusion injury [8]. Oxidative stress also acts as a signal to induce autophagy in acute ischemia-reperfusion injury [9]. Autophagy is an intracellular defense and stress regulation mechanism that works by degrading damaged organelles and denaturing long-lived proteins and nucleic acids to provide raw materials and energy for cell surviving from injury, apoptosis, and death $[10,11]$. Lower oxidative stress is very important for the activation and induction of autophagy; however, some oxidants also modify and inactivate Atg4 (autophagy-related protein 4), Atg5, LC-3 (microtubule-associated protein light chain-3), and other proteins. LC-3 is a necessary component of the autophagosome membrane and becomes involved 
TABLE 1: Effect of CQ10 on the change of SOD, GSH, TBARS, GPx, and GR in rat myocardial tissue with acute myocardial ischemiareperfusion injury.

\begin{tabular}{|c|c|c|c|c|c|c|c|c|}
\hline & \multicolumn{4}{|c|}{$\mathrm{AMI} / \mathrm{R}$} & \multicolumn{4}{|c|}{$\mathrm{CQ} 10+\mathrm{AMI} / \mathrm{R}$} \\
\hline & Sham & $2 \mathrm{~h}$ & $24 \mathrm{~h}$ & $72 \mathrm{~h}$ & Sham & $2 \mathrm{~h}$ & $24 \mathrm{~h}$ & $72 \mathrm{~h}$ \\
\hline SOD (U/mg prot & $26.89 \pm 2.42$ & $20.96 \pm 2.23$ & $15.13 \pm 1.89$ & $10.76 \pm 1.92$ & $27.29 \pm 2.14$ & $22.25 \pm 2.35^{*}$ & $18.63 \pm 2.41^{\&}$ & $14.31 \pm 1.98^{\#}$ \\
\hline $\mathrm{GSH}(\mu \mathrm{mol} / \mathrm{mg}$ protein $)$ & $5.82 \pm 0.54$ & $4.42 \pm 0.28$ & $3.13 \pm 0.33$ & $2.64 \pm 0.32$ & $5.98 \pm 0.37$ & $5.38 \pm 0.43^{*}$ & $4.27 \pm 0.37^{\&}$ & $3.47 \pm 0.41^{\#}$ \\
\hline TBARS (nmol/mg protein) & $1.33 \pm 0.09$ & $2.74 \pm 0.14$ & $3.94=$ & $5.38 \pm$ & .12 & $2.38 \pm 0.19^{*}$ & $3.19 \pm 0.31^{\&}$ & $4.57 \pm 0.42^{\#}$ \\
\hline GPx $(\mu \mathrm{mol} /$ protein $)$ & $7.41 \pm 0.64$ & $5.43 \pm 0.52$ & $3.12 \pm 0.33$ & $2.14 \pm 0.23$ & $7.38 \pm 0.56$ & $6.11 \pm 0.44^{*}$ & $4.57 \pm 0.42^{\&}$ & $3.25 \pm 0.31^{\#}$ \\
\hline GR $(\mu \mathrm{mol} /$ protein $)$ & $5.14 \pm 0.31$ & $3.28 \pm 0.34$ & $2.11 \pm 0.27$ & $1.89 \pm 0.21$ & $5.23 \pm 0.29$ & $4.56 \pm 0.38^{*}$ & $3.75 \pm 0.42^{\&}$ & $3.01 \pm 0.44^{\#}$ \\
\hline
\end{tabular}

CQ10 increased antioxidation (SOD, GSH, GPx, and GR) and reduced oxidation (TBARS) in rats with acute myocardial ischemia-reperfusion injury at same time point. SOD: superoxide dismutase; GSH: glutathione; TBARS: thiobarbituric acid reactive substances; GPx: glutathione peroxidase; GR: glutathione reductase. ${ }^{*} p<0.05$ compared with $2 \mathrm{~h}$ in AMI/R group, $n=6$; ${ }^{8} p<0.05$ compared with $24 \mathrm{~h}$ in AMI/R group, $n=6$; ${ }^{*} p<0.05$ compared with $72 \mathrm{~h}$ in AMI/ R group, $n=6$.

after activation by Atg7 and Atg3; Atg5 couples with Atg12 to help LC-3 locate on the autophagosome membrane [12-14]. Beclin-1 is a protein that interacts with either Bcl-2 or PI3k class III and plays a critical role in the regulation of both autophagy and cell death [15]. And p62, as an autophagosome-degradation marker, is a multifunctional protein located throughout the cell and involved in many signal transduction pathway [16]. Autophagy is closely linked to apoptosis. Caspase- 3 is a frequently activated death protease, catalyzing the specific cleavage of many key cellular proteins, and will be fully active under normal and apoptotic cell conditions. Caspase- 3 is activated in the apoptotic cell by both extrinsic (death ligand) and intrinsic (mitochondrial) pathways $[17,18]$. p53 protein plays a role in apoptosis, genomic stability, and inhibition of angiogenesis and initiates apoptosis [19]. They are essential proteins in the autophagy and/or apoptosis process.

Coenzyme Q10 (CQ10) is a fat-soluble quinone antioxidant with a structure that is similar to those of vitamin $\mathrm{K}$ and vitamin E. Recent research has identified CQ10 as an effective antioxidant for the prevention of oxidative damage, and it breaks down macromolecules to prevent inflammatory responses [20, 21]. Emerging research has shown that CQ10 is able to stabilize mitochondrial calcium-dependent ion channels and reduces cell energy depletion [22, 23]. However, the role of exogenous CQ10 in myocardial ischemic disease is still debatable [24-26].

Therefore, in this study, we aimed to determine if, and by what mechanism, CQ10 can ameliorate acute myocardial ischemia-reperfusion injury and improve heart function.

\section{Materials and Methods}

2.1. Animals and Drug Treatment. Adult male Sprague Dawley (SD) rats (body weight: $250 \pm 10 \mathrm{~g}$ ) were provided by the animal center of the Medical College of Xi'an Jiaotong University. Throughout the experiment, the animals were given ad libitum access to tap water.

The rats were divided into two groups: an acute myocardial ischemia-reperfusion group (AMI/R) and an acute myocardial ischemia-reperfusion with CQ10 group (CQ10 + AMI/R). The two groups were further divided into groups according to the following time points: sham, $2 \mathrm{~h}(2 \mathrm{~h}$ after reperfusion), $24 \mathrm{~h}$, and $72 \mathrm{~h}$.

In the AMI/R group, soybean oil solvent was intraperitoneally injected; in the CQ10 + AMI/R group, $5 \mathrm{~mL}$ fatsoluble CQ10 $\left(6 \mathrm{mg} \cdot \mathrm{kg}^{-1} \cdot \mathrm{mL}^{-1}\right)$ (number 24893170, Sigma, China) in soybean oil solvent was intraperitoneally injected [20]. CQ10 was consequent administered 3 days before acute ischemia and following reperfusion until the end of the experiment.

2.2. Acute Myocardial Ischemia-Reperfusion Model. The $\mathrm{AMI} / \mathrm{R}$ rat model was established as described previously [27]. SD rats were anesthetized with $2 \%$ pentobarbital (20 mg. $\mathrm{kg}^{-1}$ ) and connected to animal electrocardiography (ECG) equipment (GE Medical, Milwaukee, WI, USA). Before exposing the heart, the rats were ventilated by positive pressure using a small animal respirator (Harvard Apparatus Rodent Respirator, Harvard, USA) after tracheal intubation. The left anterior descending (LAD) coronary artery was consecutively ligated for $45 \mathrm{~min}$ before reperfusion injury, whereas in the sham group, the LAD coronary artery was not ligated but was merely wound with string. A benzylpenicillin sodium $(400000 \mathrm{U} / \mathrm{kg}$ ) injection was administered through intraperitoneal once a day for 3 days to prevent infection. Successful AMI/R operations were confirmed by ST segment-characterized ECG.

During the whole operation, the tension of the limbs, the heart rate, and autonomous respiration were monitored to assess the level of anesthesia. A mixture of pentobarbital and feed was supplied to minimize the pain caused by the procedure. As described in a previous study [23], a single injection was sufficient to keep the rats under anesthesia for the entire procedure.

At the end of the experiment, 2\% pentobarbital $\left(100 \mathrm{mg} \cdot \mathrm{kg}^{-1}\right)$ was injected into the abdomen of each rat to induce euthanasia before analysis of the AMI/R model. Eight rats in which the induction of remodeling had been successful were identified in each group. There were totally 134 rats (128 total samples) in this research for avoiding unsuccessful construction and that the same one could not be used in various detecting methods, such as echocardiography, hemodynamics, myocardial staining, histopathology, and immunohistochemistry. 


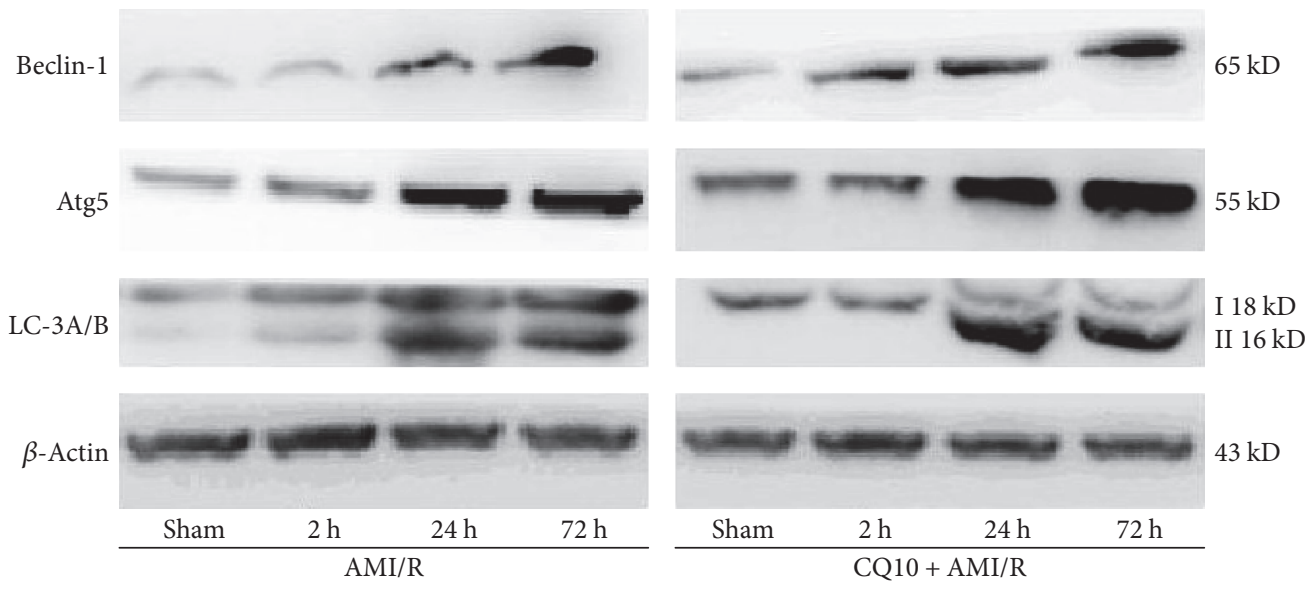

(a)

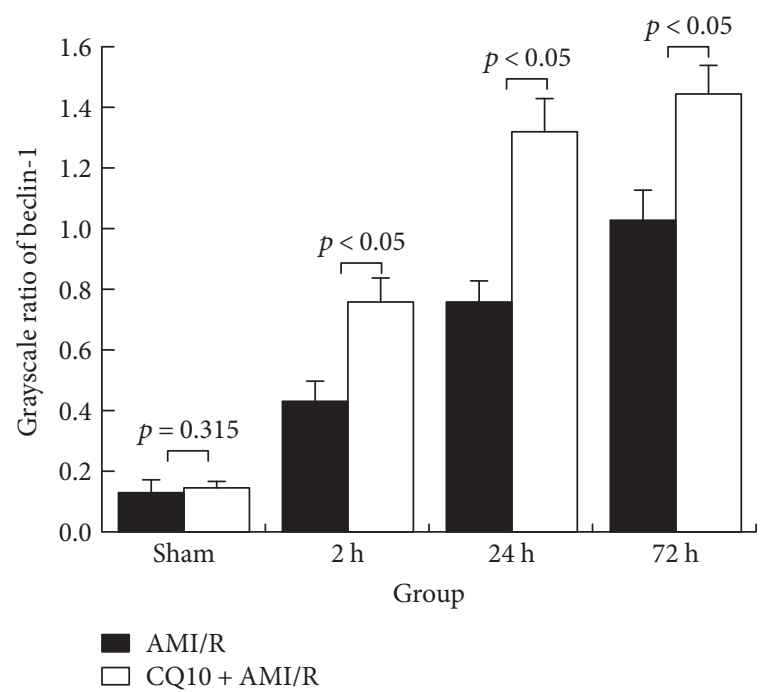

(b)

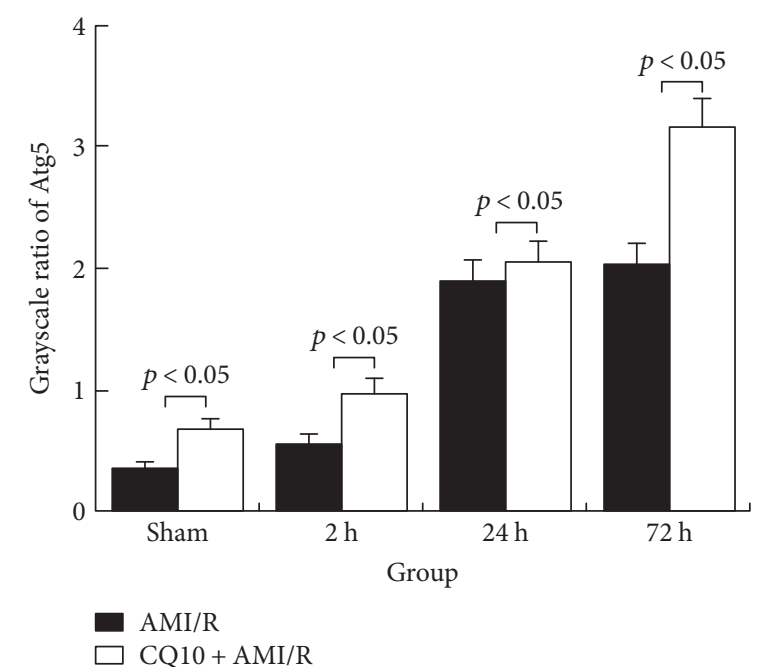

(c)

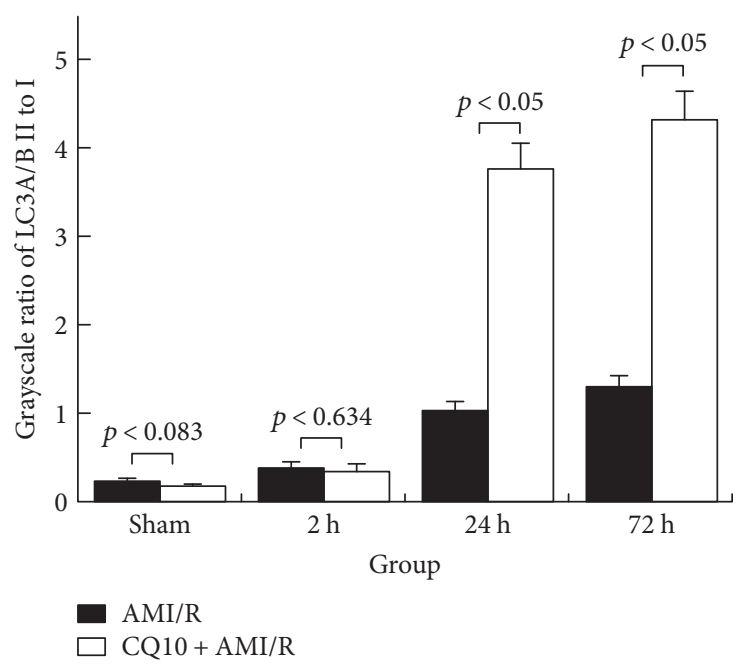

(d)

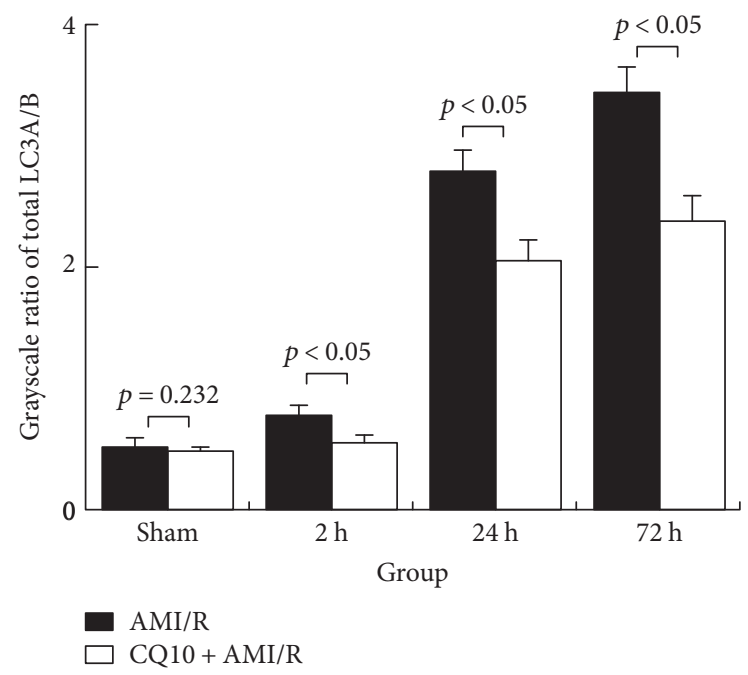

(e)

Figure 1: CQ10 enhanced autophagy in rats with acute myocardial ischemia-reperfusion injury. (a) The grayscale of beclin-1, Atg5 LC-3A/B, and $\beta$-actin was detected by Western blot. (b and c) CQ10 increased autophagy beginning protein beclin-1 and Atg5 expression at same time point. ( $\mathrm{d}$ and e) CQ10 increased autophagy activity and improve the transformation of LC-3A/B II to I. $p<0.05$ was considered statistically significant, $n=6$. 


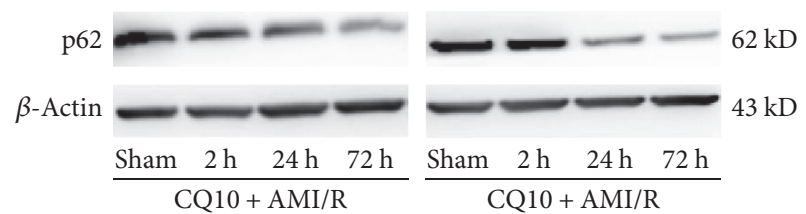

(a)

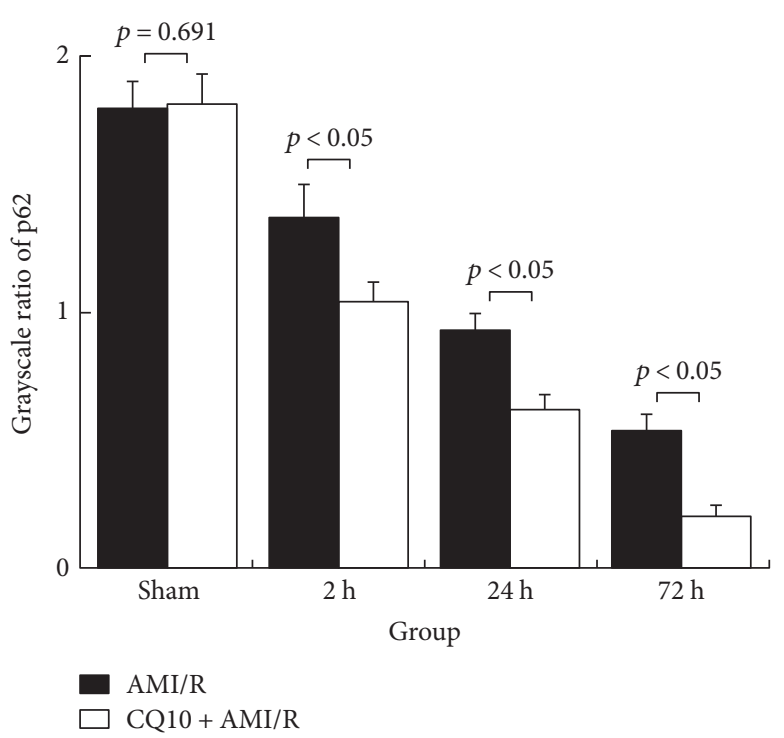

(b)

FIGURE 2: CQ10 decreased p62 protein expression (as grayscale ratio of $\mathrm{p} 62$ to $\beta$-actin) in rats with acute myocardial ischemia-reperfusion injury. (a) The grayscale of autophagy-negative protein p62 expression was detected by Western blot. (b) CQ10 decreases p62 protein expression at same time point. $p<0.05$ was considered statistically significant, $n=6$.

\subsection{Echocardiography and Hemodynamic Assessment of} Cardiac Function. The structure and heart function of each subject were evaluated by the length of the $10 \mathrm{~S}$ probe shaft and the use of an M-type two-dimensional ultrasound instrument (Sequoia 512, Siemens AG, Germany), respectively. Three consecutive cardiac cycles were captured from at least six subjects from each group by digital image analysis software. Subsequently, a sidearm of the aortic cannula was inserted into the left ventricle from the right carotid artery and was connected to a pressure transducer (Millar Instruments, Houston, Texas, USA). The organism function experimental system (type number BL-420F, Texas, USA) was used to amplify the signal and analyze the data. Myocardial tissue was preserved for the subsequent step.

2.4. Western Blotting Analysis. Equal amounts of proteins from each sample were separated with Any-kD sodium dodecyl sulfate (SDS) precast gel. After blocking with 5\% nonfat milk, polyvinylidene fluoride membranes were hybridized with the following antibodies: monoclonal beclin-1 antibody at $1: 1000$ dilution, monoclonal Atg5 antibody at $1: 1000$ dilution, monoclonal LC-3A/B antibody at $1: 1000$ dilution, monoclonal p53 antibody at $1: 1000$ dilution, and monoclonal caspase- 3 antibody at 1:1000 dilution. The membranes were subsequently hybridized with the corresponding secondary antibodies. $\beta$-Actin proteins were used as loading and internal controls. The data are shown as the grayscale ratio of the target protein to $\beta$-actin.

2.5. Myocardial Tissue Staining. Each myocardial tissue sample was cut transversely. Myocardial pathology was observed by hematoxylin and eosin (HE) staining and Masson trichrome staining. Myocardial ischemic risk areas were determined as described previously [28]. To delineate the ischemic area-at-risk, $1 \%$ Evans blue $(1 \mathrm{~mL})$ was injected following the occlusion of the coronary artery at the level of LAD ligation. The whole heart was then removed and transected parallel to the atrioventricular groove at the center of the infarct area in 1\% triphenyl tetrazolium chloride (TTC) solution for $10 \mathrm{~min}$. There were at least six rats in each group and three sections per rat. The observer was blinded to the animal treatment groups when evaluating myocardial infarct sizes.

For immunofluorescence staining, 4',6-diamidino-2-phenylindole (DAPI), Bax antibody, Bak antibody, and the corresponding fluorescent secondary antibodies were used. Immunofluorescence staining was quantified by Image-Pro Plus software. In the AMI/R rats, the DAPI, Bax, or Bak fluorescence density values of the sham group were set to 100 standard density; other groups were represented by fluorescence density values relative to the standard density.

2.6. Measurement of Oxidants and Antioxidants. Heart tissue was weighed and homogenized in ice-cold normal saline. Heart homogenate was mixed with an equal volume of cold Tris-ethylenediaminetetraacetic acid (EDTA). The separated cytosolic fraction was used for the subsequent measurement.

Oxidant levels were measured using a thiobarbituric acid reactive substances (TBARS) assay kit (Cayman Chemical Company, Ann Arbor, USA).

The levels of the antioxidants glutathione peroxidase $(\mathrm{GPx})$, glutathione reductase (GR), glutathione (GSH), and superoxide dismutase (SOD) were measured in each group according to the instructions provided by the manufacturers of the GPx assay kit (Jining, Shanghai, China), the GR assay kit (Yueyan Biological Technology Co. Ltd., Shanghai, China), the GSH assay kit (Jiancheng, Nanjing, China), and the SOD assay kit (Dojindo, Shanghai, China), respectively. 


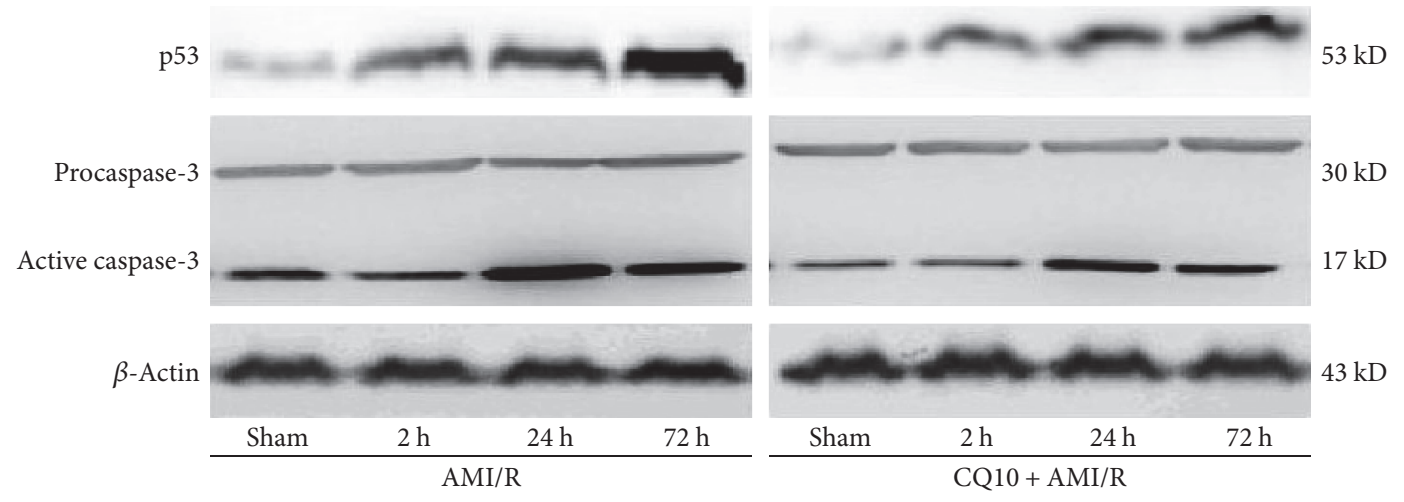

(a)

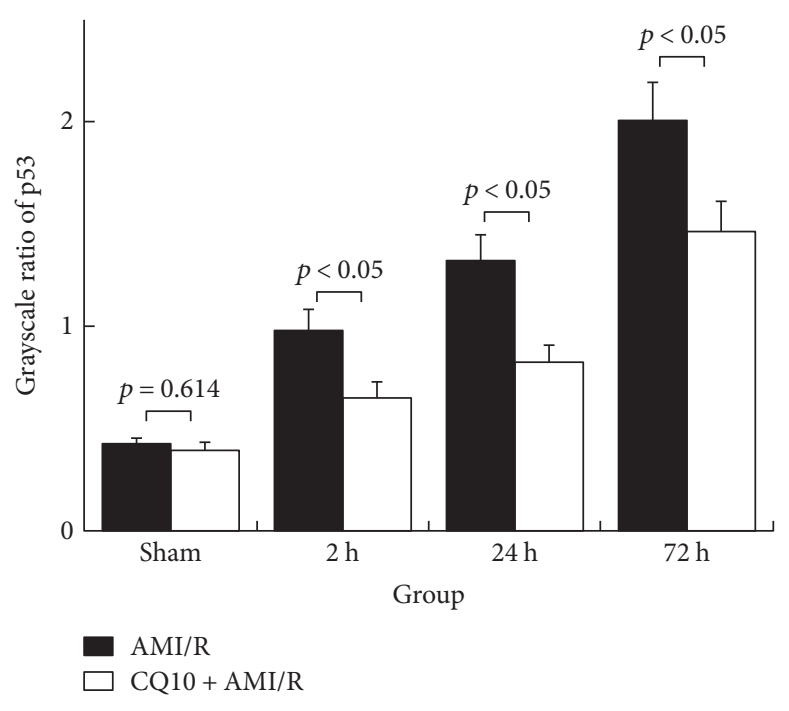

(b)

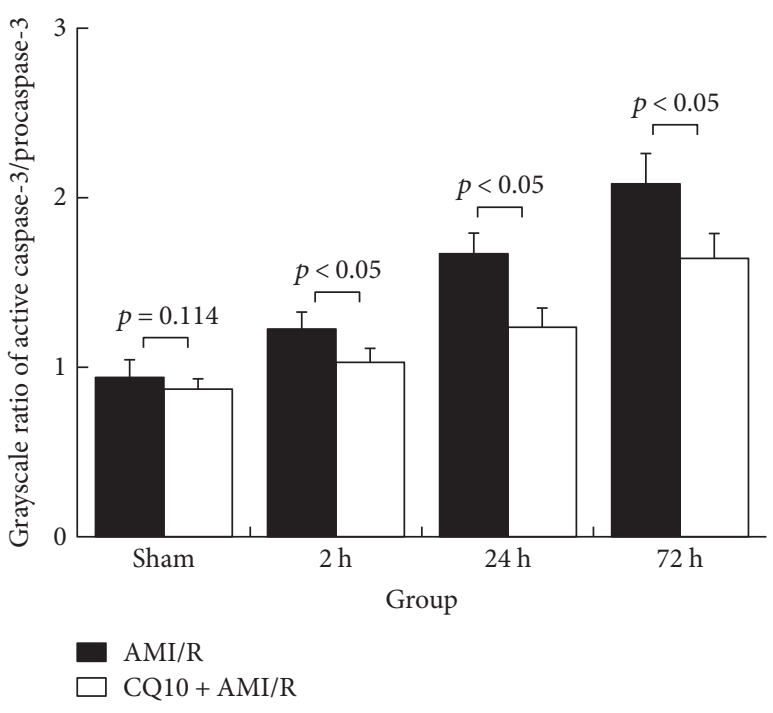

(c)

Figure 3: (a) p53 and caspase-3 expression (as grayscale ratio of p53 or caspase-3 to $\beta$-actin) in rats with acute myocardial ischemiareperfusion injury was shown. (b and c) CQ10 decreases apoptosis-associated protein expression of p53 and caspase-3. Caspase-3 activity was shown as a grayscale ratio of active caspase- 3 to procaspase-3. $p<0.05$ was considered statistically significant, $n=6$.

2.7. Detection of Cellular Energy Supply. The ATPlite Luminescence Assay System (PerkinElmer, USA) was used to evaluate cellular energy supply according to manufacturer's instructions. Data are shown as ATP concentrations relative to the ATPlite Assay System standard sample.

2.8. Statistical Analysis. The random variables follow a normal distribution in this analysis. All data are presented as mean \pm standard, and $p<0.05$ was considered statistically significant. Analysis of variance (ANOVA) was used to examine differences between the two groups, followed by either the least significant difference (LSD) test or the Games-Howell test according to the homogeneity of variance.

2.9. Compliance with Ethical Standards. The animal protocol was approved by the Xi'an Jiaotong University laboratory animal ethics review board (number A-2012-975SL), and the animal procedures conformed with the guidelines provided by Directive 2010/63/EU of the European
Parliament on the protection of animals used for scientific purposes.

\section{Results}

3.1. CQ10 Reduced Oxidative Stress in Rats with Acute Myocardial Ischemia-Reperfusion Injury. Because CQ10 is a well-known antioxidant agent, we demonstrated that preconditioning with CQ10 significantly increased antioxidant levels of GPx, GR, SOD, and GSH $(p<0.05, n=6)$ and decreased oxidant TBARS levels $(p<0.05, n=6)$ in rats with acute myocardial ischemia-reperfusion injury (Table 1).

3.2. CQ10 Enhanced Autophagy in Rats with Acute Myocardial Ischemia-Reperfusion Injury. We used Western blotting detection to demonstrate that CQ10 noticeably increased the levels of the positively associated autophagy proteins beclin-1 and Atg5 at the same time points of acute myocardial ischemia-reperfusion injury $(p<0.05, n=6)$. Furthermore, the ratio of LC-3II to LC-3I as a standard of 
$\mathrm{AMI} / \mathrm{R}$
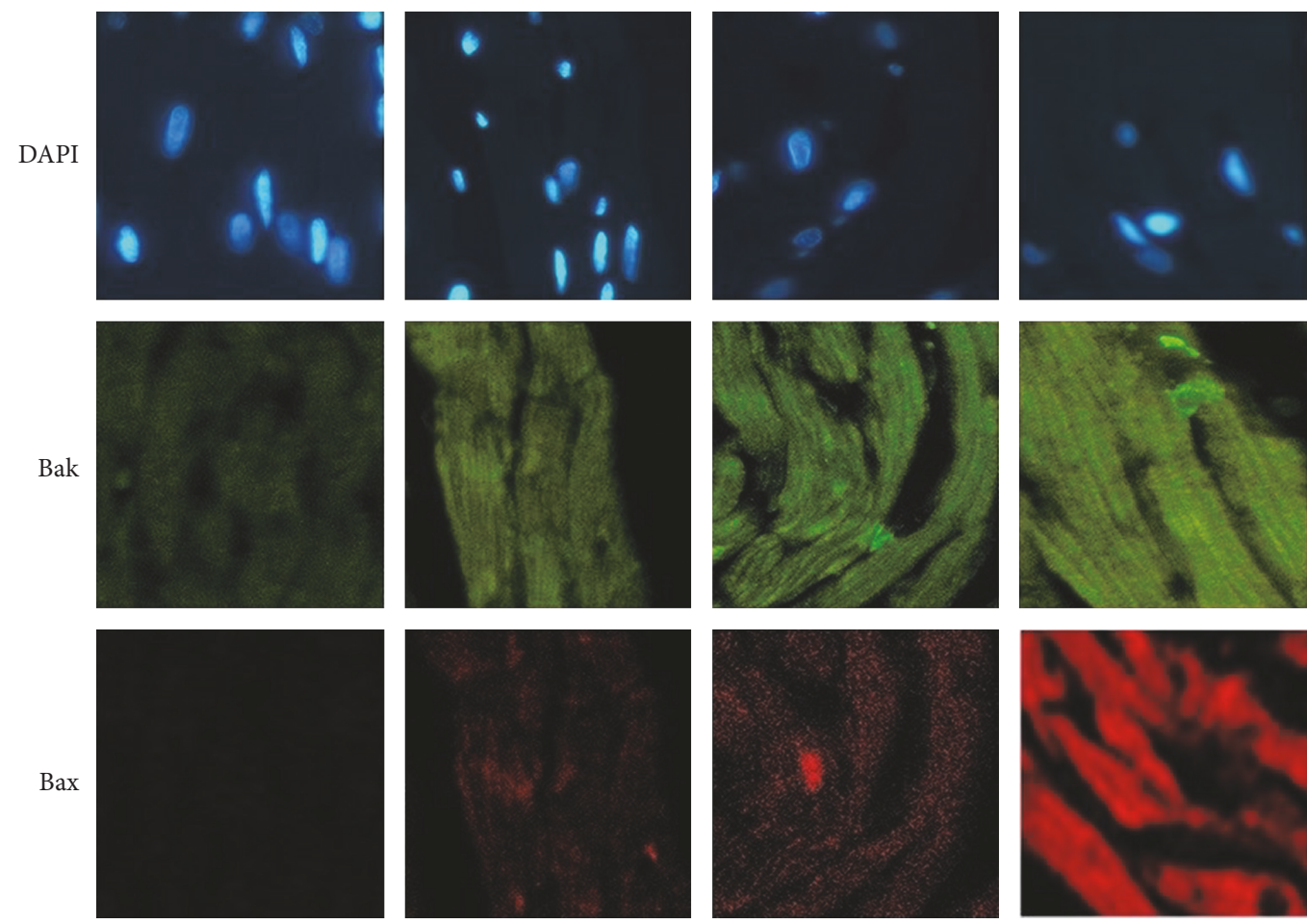

$24 \mathrm{~h}$

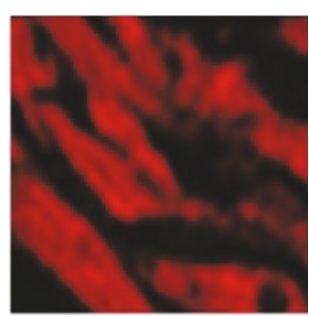

Sham

$2 \mathrm{~h}$

$72 \mathrm{~h}$

(a)
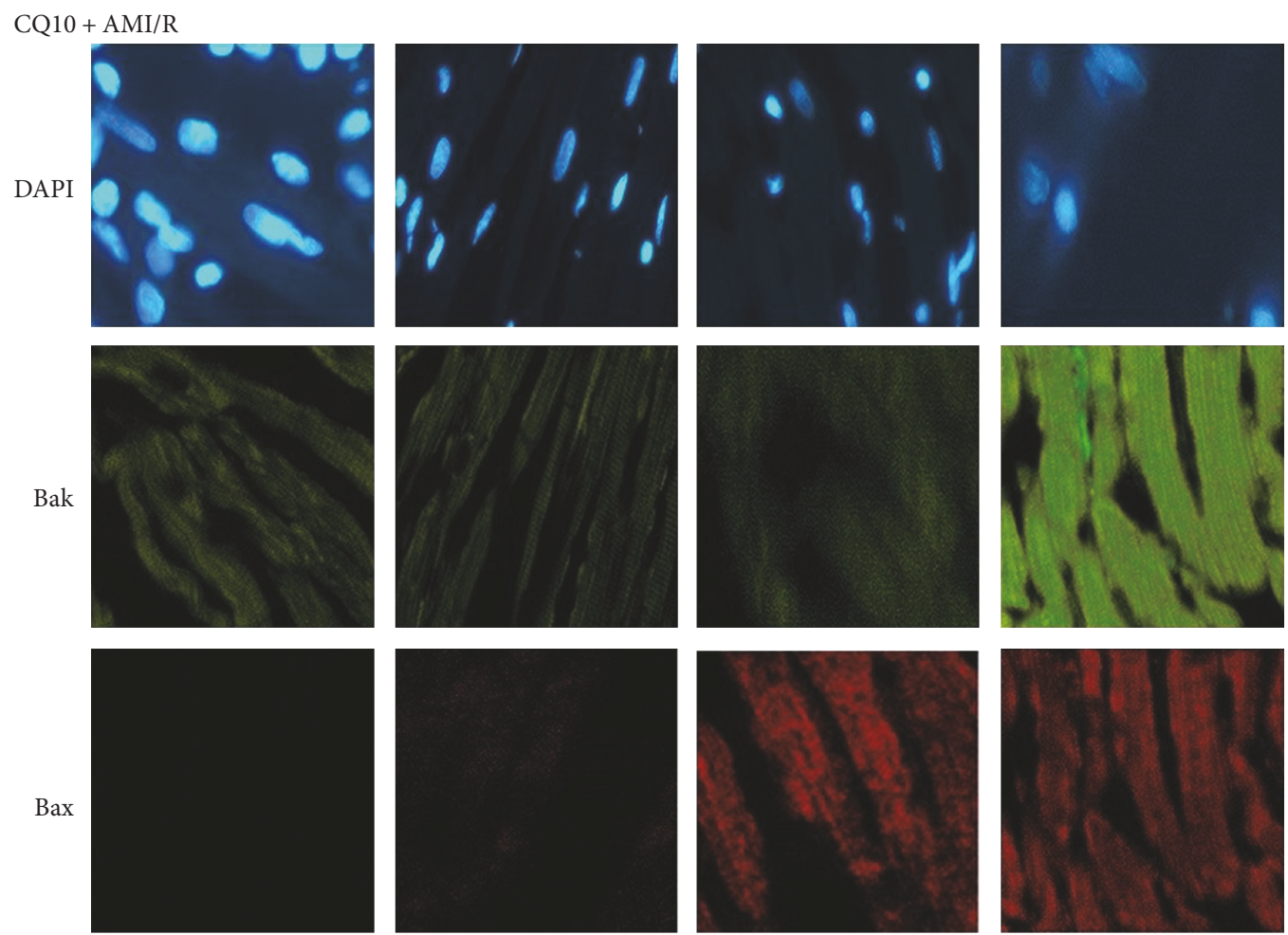

$2 \mathrm{~h}$

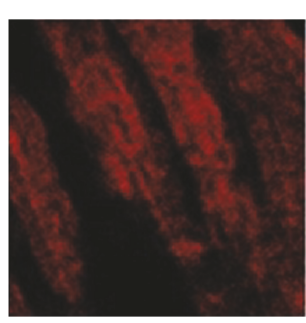

$24 \mathrm{~h}$

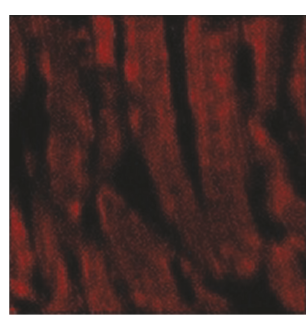

$72 \mathrm{~h}$

(b)

FIGURE 4: DAPI/Bak/Bax immunofluorescence staining in rats with acute myocardial ischemia-reperfusion injury $(\times 400)$. (a) DAPI/Bak/Bax immunofluorescence staining in different time of AMI/R group. (b) DAPI/Bak/Bax immunofluorescence staining in different time of $\mathrm{AMI} / \mathrm{R}+\mathrm{CQ} 10$ group. 


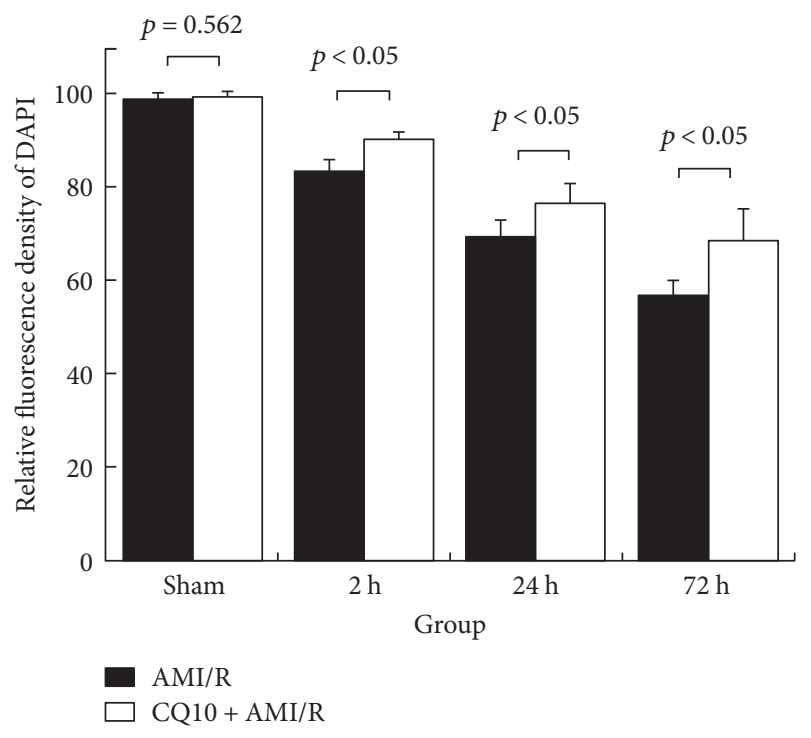

(a)

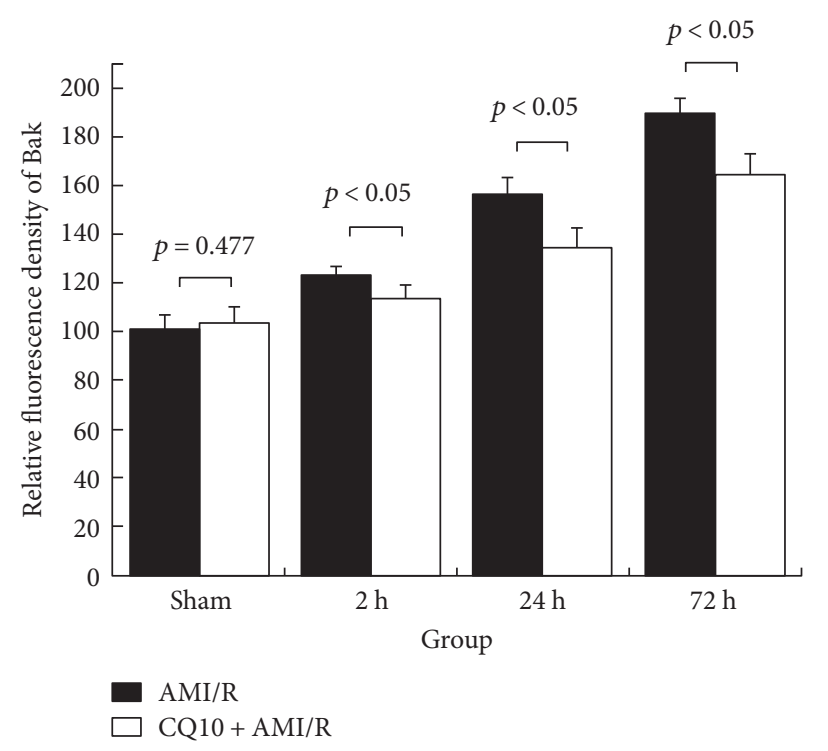

(b)

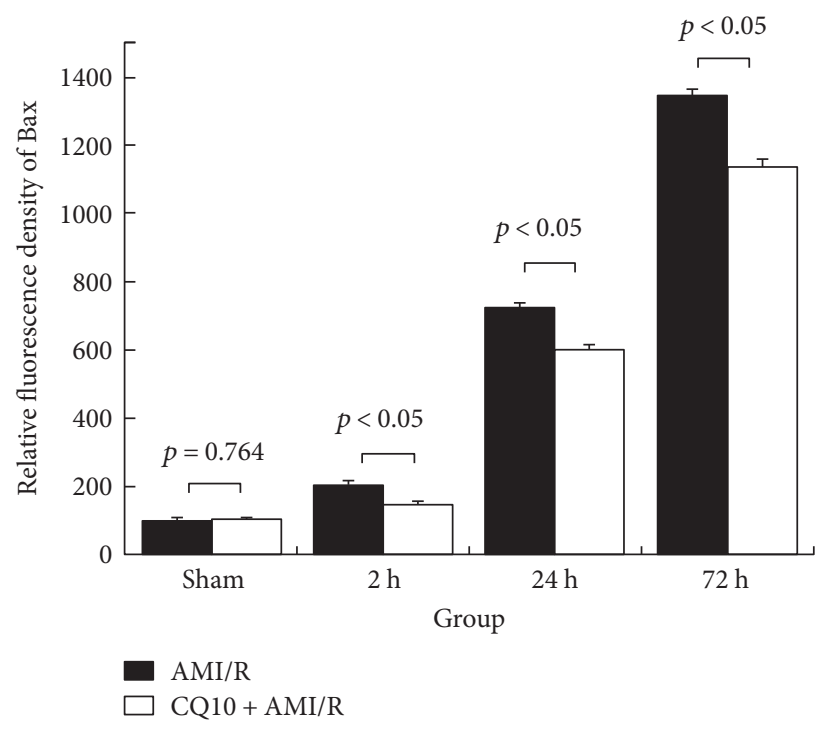

(c)

FIGURE 5: Relative fluorescence density of DAPI/Bak/Bax in rats with acute myocardial ischemia-reperfusion injury. Sham group's DAPI, Bax, or Bak fluorescence density was set as 100 standard density. Other groups' DAPI, Bax, or Bak fluorescence density was present as relative fluorescence density compared with sham group. Six samples were selected from each group, and three horizons were observed in the ischemic penumbra of each sample $(\times 400)$. (a) CQ10 increases DAPI fluorescence density. (b) CQ10 decreases Bax fluorescence density. (c) CQ10 decreases Bak fluorescence density. $p<0.05$ was considered statistically significant.

autophagy activity was clearly increased by CQ10 at the same time points as acute myocardial ischemia-reperfusion injury (Figure $1 ; p<0.05, n=6$ ). However, the total LC-3 and negatively associated autophagy protein p62 levels were significantly reduced in the CQ10 group compared with the $\mathrm{AMI} / \mathrm{R}$ group at the same time points in (Figure $2 ; p<0.05, n=6$ ).

\subsection{CQ10 Reduced Proapoptotic Proteins in Rats with Acute} Myocardial Ischemia-Reperfusion Injury. We also proved that CQ10 reduced proapoptotic protein p53, and the ratio of active caspase- 3 to procaspase- 3 was significantly higher at $2 \mathrm{~h}, 24 \mathrm{~h}$, and $72 \mathrm{~h}$ in rats with acute myocardial ischemia-reperfusion injury than in the control rats (Figure 3; $p<0.05, n=6$ ).

DAPI fluorescence revealed the number of intact nuclei, but did not show myocardial necrosis due to DNA and RNA condensation, fission, or burst. Compared with the AMI/R group, there were more myocardial nuclei in the CQ10 group at the same time points. Bak/Bax fluorescence expression was stronger in the CQ10 group than in the AMI/R group at the same time points (Figures 4 and $5 ; p<0.05$ ).

3.4. CQ10 Preserved Myocardial Activity in Rats with Acute Myocardial Ischemia-Reperfusion Injury. HE and Masson staining facilitated the visualization of cardiac structural 

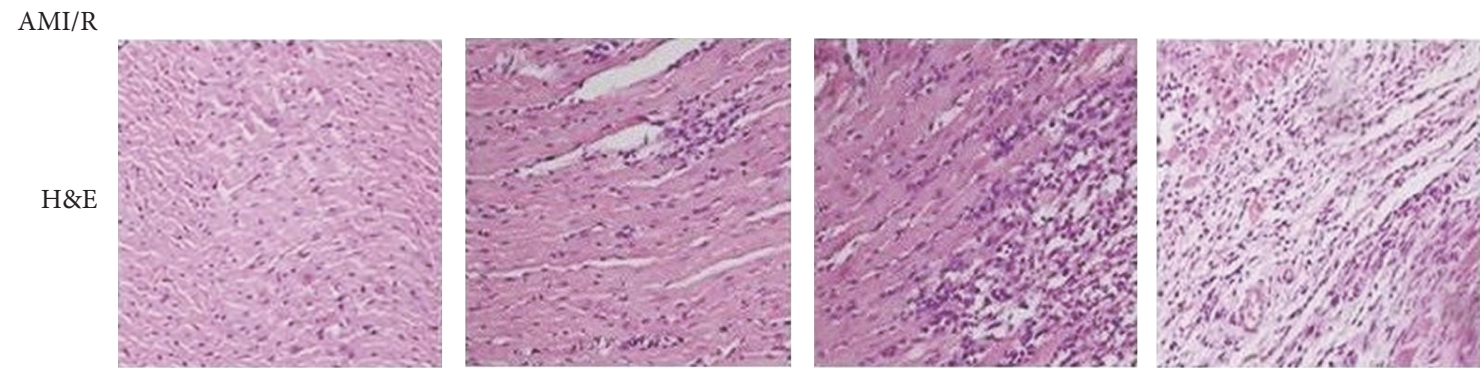

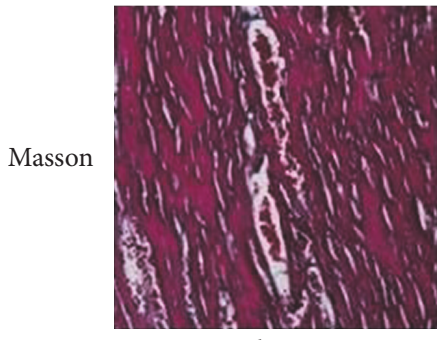

Sham

$\mathrm{CQ} 10+\mathrm{AMI} / \mathrm{R}$

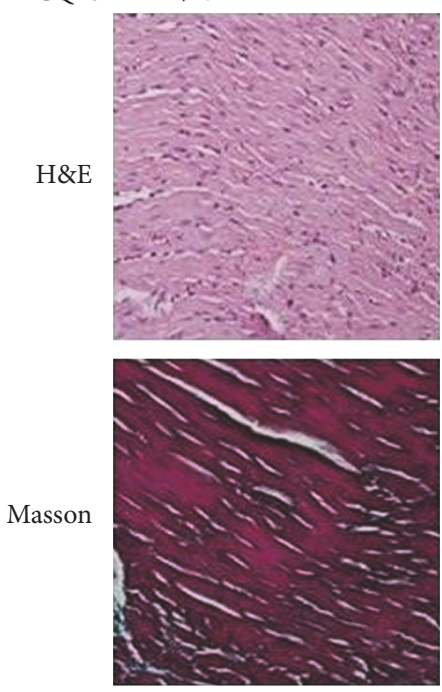

Sham

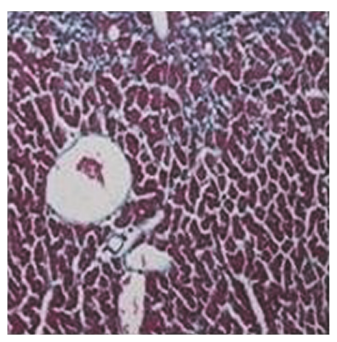

$2 \mathrm{~h}$

(a)
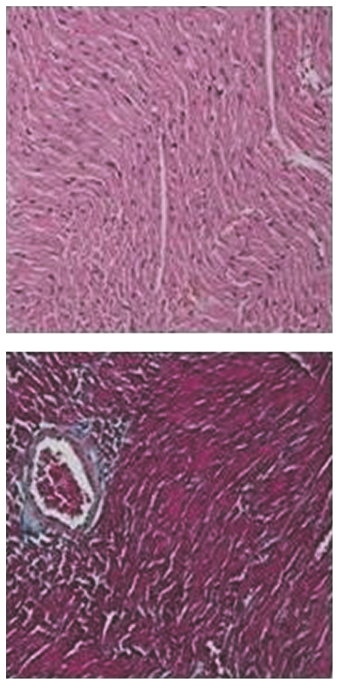

$2 \mathrm{~h}$

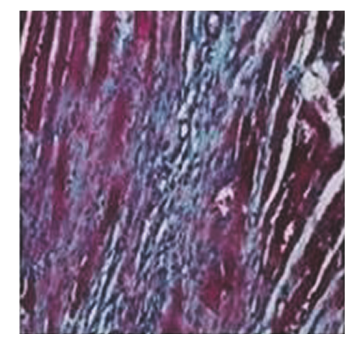

$24 \mathrm{~h}$
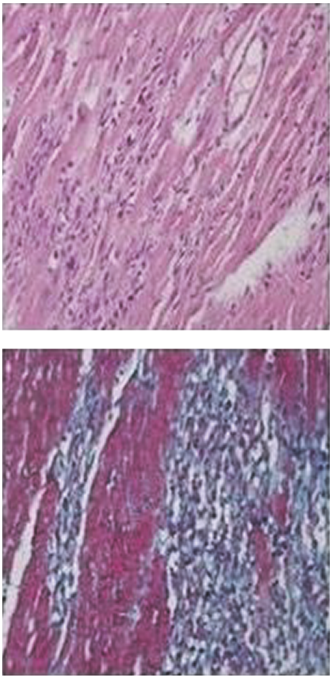

$24 \mathrm{~h}$

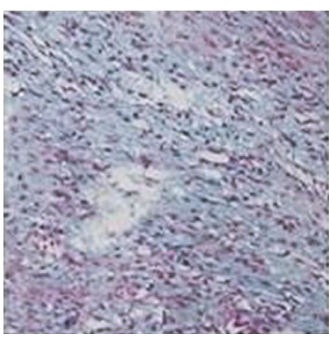

$72 \mathrm{~h}$
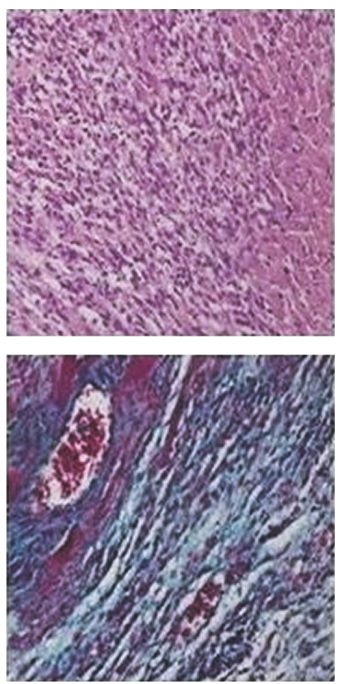

$72 \mathrm{~h}$

(b)

Figure 6: CQ10 could delay myocardial fibrosis, denaturation, and necrosis in rats with acute myocardial ischemia-reperfusion injury by $\mathrm{H} \& \mathrm{E}$ and Masson staining $(\times 100)$. (a) H\&E and Masson staining in different time of AMI/R group. (b) H\&E and Masson staining in different time of AMI/R + CQ10 group.

disorder, myocardial cell denaturation and necrosis, and fibrous scar tissue formation in rats with acute myocardial ischemia-reperfusion injury. However, in the ischemic penumbra of the CQ10 group, these phenomena were not as serious as in the AMI/R group (Figure 6).

Infarct size was estimated as a percentage of the ischemic area-at-risk. At $2 \mathrm{~h}, 24 \mathrm{~h}$, and $72 \mathrm{~h}$ following acute myocardial ischemia-reperfusion injury, CQ10 significantly reduced infarct size (Figure $7 ; p<0.05, n=6$ ).

3.5. CQ10 Improved Cardiac Function in Rats with Acute Myocardial Ischemia-Reperfusion Injury. We used echocardiography and hemodynamic analysis to investigate cardiac function during acute myocardial ischemia-reperfusion injury. There was no difference between the two groups with regard to the following parameters: diastolic interventricular septal wall thickness (IVSd), systole interventricular septal wall thickness (IVS), diastolic left ventricular internal dimension (LVIDd), systole left ventricular internal dimension (LVID), and systole volume (SV), which represented cardiac structure and size at the same average heart rate $(p>0.05$, $n=6)$. From $2 \mathrm{~h}$ after reperfusion, CQ10 preconditioning improved cardiac systolic and diastolic functions (left ventricular ejection fraction (EF), left ventricular systolic pressure (LVSP), and left ventricular systolic pressure (LVEDP), the maximum change rate of left ventricular pressure rise 

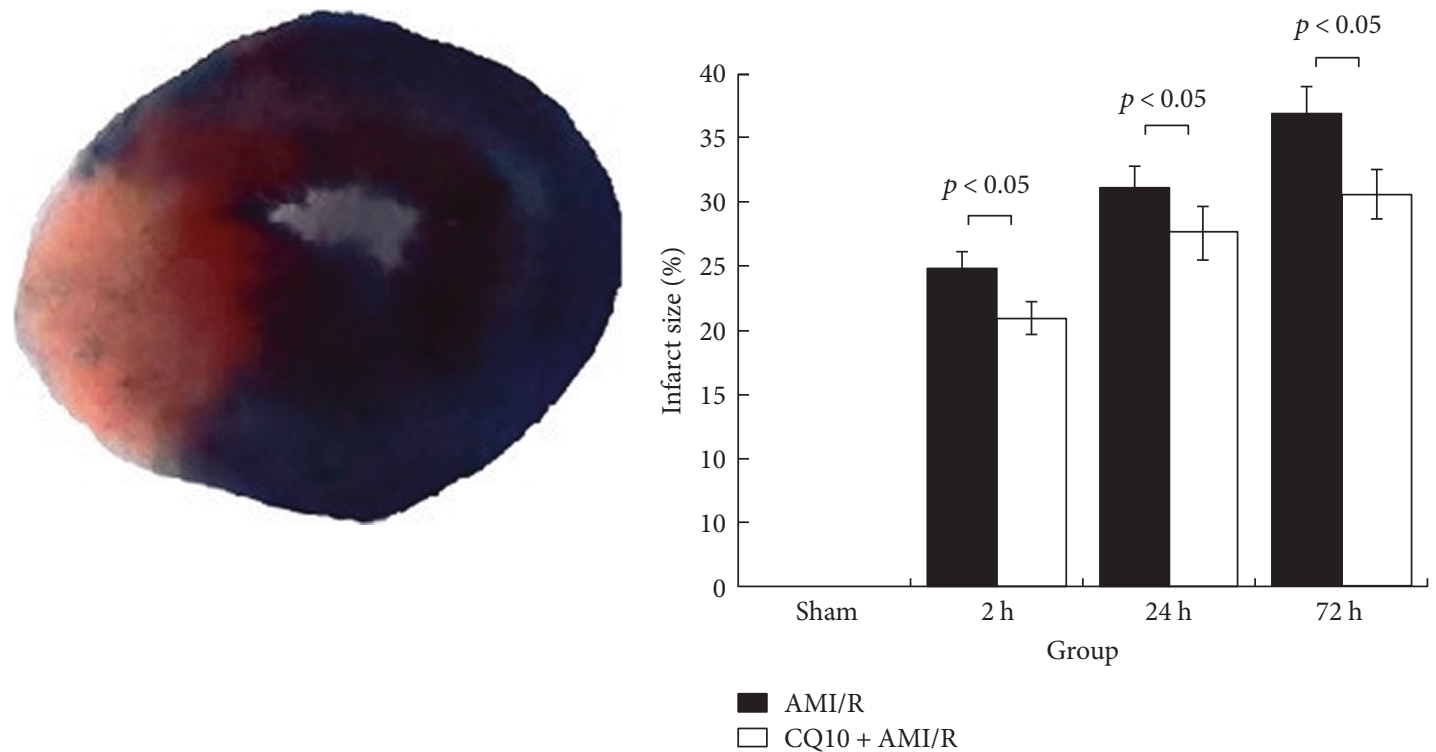

(a)

(b)

FIGURE 7: CQ10 could reduce infarct size (as a percentage of the ischemic area-at-risk) in rats with acute myocardial ischemia-reperfusion injury by TTC staining. (a) Infarct area appeared khaki, whereas the viable myocardium was red by TTC staining. (b) CQ10 could reduce the percentage of the ischemic area-at-risk. $p<0.05$ was considered statistically significant, $n=6$.

TABLE 2: The change of cardiac function.

\begin{tabular}{|c|c|c|c|c|c|c|c|c|}
\hline & \multicolumn{4}{|c|}{$\mathrm{AMI} / \mathrm{R}$} & \multicolumn{4}{|c|}{ CQ10 + AMI/R } \\
\hline & Sham & $2 \mathrm{~h}$ & $24 \mathrm{~h}$ & $72 \mathrm{~h}$ & Sham & $2 \mathrm{~h}$ & $24 \mathrm{~h}$ & $72 \mathrm{~h}$ \\
\hline HR (bpm) & $\begin{array}{l}395.26 \\
\pm 35.78\end{array}$ & $\begin{array}{l}396.14 \\
\pm 42.74\end{array}$ & $\begin{array}{l}402.20 \\
\pm 42.12\end{array}$ & $\begin{array}{l}418.54 \\
\pm 39.56\end{array}$ & $\begin{array}{l}112.70 \\
\pm 39.34\end{array}$ & $\begin{array}{l}397.24 \\
\pm 36.24\end{array}$ & $\begin{array}{l}409.20 \\
\pm 38.92\end{array}$ & $\begin{array}{l}392.87 \\
\pm 42.13\end{array}$ \\
\hline IVSd (mm) & $1.64 \pm 0.27$ & $1.58 \pm 0.34$ & $1.62 \pm 0.31$ & $1.61 \pm 0.32$ & $1.64 \pm 0.34$ & $1.72 \pm 0.28$ & $1.65 \pm 0.41$ & $1.68 \pm 0.39$ \\
\hline IVS (mm) & $2.56 \pm 0.37$ & $2.30 \pm 0.44$ & $2.55 \pm 0.81$ & $2.49 \pm 0.44$ & $2.67 \pm 0.13$ & $2.47 \pm 0.58$ & $2.55 \pm 0.18$ & $2.43 \pm 0.21$ \\
\hline LVIDd (mm) & $4.11 \pm 0.94$ & $4.18 \pm 1.48$ & $4.15 \pm 0.67$ & $4.21 \pm 0.67$ & $4.15 \pm 0.19$ & $3.97 \pm 0.16$ & $4.05 \pm 0.45$ & $4.14 \pm 0.42$ \\
\hline LVID (mm) & $2.45 \pm 0.57$ & $2.50 \pm 0.95$ & $2.53 \pm 0.79$ & $2.43 \pm 0.89$ & $2.53 \pm 0.18$ & $2.57 \pm 0.12$ & $2.45 \pm 0.27$ & $2.49 \pm 0.38$ \\
\hline $\mathrm{SV}\left(\mathrm{cm}^{3}\right)$ & $0.25 \pm 0.08$ & $0.24 \pm 0.05$ & $0.23 \pm 0.04$ & $0.23 \pm 0.06$ & $0.22 \pm 0.07$ & $0.24 \pm 0.06$ & $0.20 \pm 0.08$ & $0.23 \pm 0.10$ \\
\hline FS (\%) & $53.17 \pm 5.76$ & $54.74 \pm 1.87$ & $55.45 \pm 9.63$ & $52.37 \pm 4.13$ & $52.12 \pm 4.82$ & $50.13 \pm 2.07$ & $54.17 \pm 7.88$ & $51.21 \pm 4.37$ \\
\hline $\mathrm{EF}(\%)$ & $93.25 \pm 3.14$ & $80.23 \pm 7.12$ & $70.26 \pm 5.26$ & $59.12 \pm 5.81$ & $92.14 \pm 2.65$ & $85.42 \pm 5.27^{*}$ & $79.56 \pm 5.36^{*}$ & $67.12 \pm 6.18^{*}$ \\
\hline $\begin{array}{l}\text { LVSP } \\
(\mathrm{mmHg})\end{array}$ & $84.25 \pm 12.85$ & $74.67 \pm 10.15$ & $65.84 \pm 9.84$ & $61.84 \pm 10.04$ & $84.42 \pm 12.55$ & $79.45 \pm 10.25^{*}$ & $71.44 \pm 14.13^{*}$ & $67.82 \pm 13.32^{*}$ \\
\hline $\begin{array}{l}\text { LVEDP } \\
(\mathrm{mmHg})\end{array}$ & $2.16 \pm 0.09$ & $2.53 \pm 0.08$ & $2.86 \pm 0.12$ & $3.42 \pm 0.07$ & $2.19 \pm 0.14$ & $2.33 \pm 0.09^{*}$ & $2.64 \pm 0.17^{*}$ & $2.97 \pm 0.15^{*}$ \\
\hline $\begin{array}{l}+\mathrm{dp} / \mathrm{dtmax} \\
(\mathrm{mmHg} / \mathrm{ms})\end{array}$ & $3.79 \pm 0.12$ & $3.37 \pm 0.06$ & $2.18 \pm 0.07$ & $1.84 \pm 0.08$ & $3.80 \pm 0.08$ & $3.49 \pm 0.06$ & $2.88 \pm 0.09^{*}$ & $2.25 \pm 0.12^{*}$ \\
\hline $\begin{array}{l}-\mathrm{dp} / \mathrm{dtmax} \\
(\mathrm{mmHg} / \mathrm{ms})\end{array}$ & $3.02 \pm 0.06$ & $2.56 \pm 0.48$ & $2.37 \pm 0.54$ & $1.57 \pm 0.54$ & $2.92 \pm 0.08$ & $2.75 \pm 0.04^{*}$ & $2.49 \pm 0.09^{*}$ & $1.89 \pm 0.09^{*}$ \\
\hline
\end{tabular}

CQ10 improved cardiac function but did not change cardiac structure in rats with acute myocardial ischemia-reperfusion injury at same time point. IVSd: diastolic interventricular septal wall thickness; IVS: systole inter-ventricular septal wall thickness; LVIDd: diastolic left ventricular internal dimension; LVID: systole left ventricular internal dimension; EF: left ventricular ejection fraction; SV: systole volume; FS: left ventricular fractional shortening; LVSP: left ventricular systolic pressure; LVEDP: left ventricular end-diastolic pressure; $+\mathrm{dp} / \mathrm{dtmax}$ : the maximum change rate of left ventricular pressure rise; $-\mathrm{dp} /$ dtmax: the maximum change rate of left ventricular pressure fall; AMI/R: acute myocardial ischemia/reperfusion. ${ }^{*} p<0.05$ was considered statistically significant; $n=6$.

$(+\mathrm{dp} / \mathrm{dtmax})$, and the maximum change rate of left ventricular pressure fall $(-\mathrm{dp} / \mathrm{dtmax})$ compared with the AMI/R group (Table $2 ; p<0.05, n=6)$ ).
3.6. CQ10 Increased Direct Energy Supply in Rats with Acute Myocardial Ischemia-Reperfusion Injury. Finally, the relative ATP concentration, which is representative of direct energy 


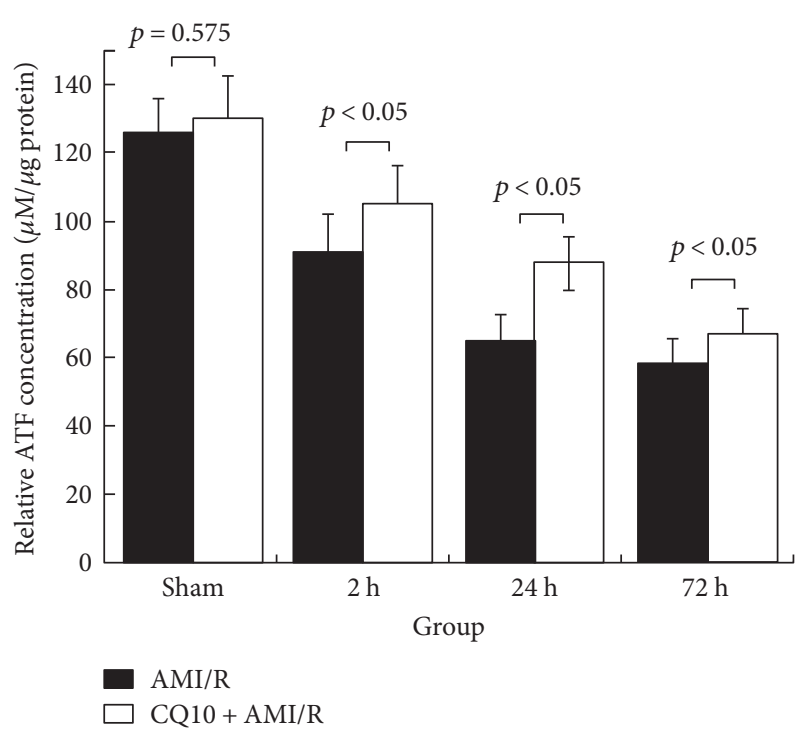

FIGURE 8: CQ10 increased relative ATP concentration in rats with acute myocardial ischemia-reperfusion injury. Date was shown as relative ATP concentration according to ATPlite Assay System standard sample. $p<0.05$ was considered statistically significant, $n=6$.

supply, was increased by CQ10 during acute myocardial ischemia-reperfusion injury (Figure 8).

\section{Discussion}

It has become increasingly clear that the level of oxidants is greatly increased in the postischemic heart, and oxidants play a critical role in postischemic injury. Lipid peroxidation, induced by oxygen radicals, results in cell membrane breakdown, which leads to inhibition of the sodium-potassium ATPase, cell swelling, and mitochondrial dysfunction [6]. As previously reported, CQ10 plays a key role in the mitochondrial respiratory chain by preventing oxidative stress injury to the mitochondrial membrane $[29,30]$. It is worth noting that there is a synergistic effect between Bax and Bak on mitochondrial fragmentation and perforation, cell apoptosis, and programmed cell death. In our research, both the balance between antioxidants (GPx, GR, SOD, and GSH) and oxidants and the levels of Bax and Bak were regulated by CQ10. Furthermore, the ATP concentration increased after CQ10 preconditioning for direct energy supply.

Autophagy is a widespread self-ingesting phenomenon that normally takes place in the biosphere at a low level. It is considered to be a protection mechanism that acts by degrading impaired proteins and/or organelles. It ensures that the cells survive with adequate supplies of materials and energy through recycling under most conditions of stress, such as acute ischemia and reperfusion, chemical toxicity, and oxidation [31-33]. However, high levels of oxidants are responsible for the inactivity of Atg5, beclin-1, and other autophagy-associated proteins $[12,14,34]$. Other studies have also proved that preconditioning with autophagyinducing agents protects myocardial cells after acute ischemia-reperfusion [34]. In our study, Atg5, beclin-1, and the ratio of LC-3II to LC3-I, which represent autophagy activity, are increased after acute ischemia-reperfusion injury with CQ10 preconditioning. The study also demonstrates that CQ10 reduces total LC-3 levels and p62.

Emerging evidence suggests interactions among the crucial proteins of autophagy and apoptosis as follows: beclin1 binding to Bcl-2 family, caspase- 3 cleaving beclin-1, [35], p53 promoting the proapoptotic proteins and inhibiting Bcl-2, and p53 posttranscriptionally downregulating LC-3 and controlling autophagic flux [36]. In our study, apoptosis protein levels are reduced by CQ10 preconditioning. Both proapoptosis protein and apoptosis protein of $\mathrm{Bcl}-2$ family have taken important roles in cell apoptosis process. Cell intact nuclei were stained by DAPI. Bak and Bax were located in the mitochondrial outer membrane by immunofluorescence staining. Bax and Bak have a collaborative effect to increase the mitochondrial outer membrane permeability and aggravate cell death. As previously informed, p53 and caspase- 3 are also wildly proved proapoptosis effect in many stress condition.

In all, antioxidative function, energy survival, and autophagy activation are the major causes of infarct size reduction and cardiac function improvement after acute ischemia-reperfusion injury with CQ10 preconditioning. But timely reperfusion of the acute ischemic myocardium is essential for myocardial salvage. A number of mechanisms have been proposed to explain the mediation of reperfusion injury. These include cellular calcium loading, endothelium cell swelling, impaired vascular relaxation, and the action of oxygen radicals [3-6]. The impact of CQ10 is actually restricted to delaying the changes after ischemia-reperfusion injury, not abolishing anything $[15,24]$. In clinical situations, the outcomes for acute coronary syndrome patients who are suffering from ischemia-reperfusion are influenced by many unpredictable events, such as myocardial stunning, coronary vascular spasms, or lethal arrhythmia. Further research is required $[24,25]$.

For some limitation, this study just focuses on short period of outcome after ischemia-reperfusion injury. In order to sufficiently evaluate whether CQ10 preconditioning reduces or only delays the detrimental effect of ischemiareperfusion injury, a research designed for different doses of CQ10 and a long of outcome after ischemia-reperfusion injury is needed.

In summary, we have established that CQ10 preconditioning regulates the balance of antioxidants and oxidants, enhances autophagy levels and activity, reduces myocardial apoptosis, and improves cardiac function in rats with acute ischemia-reperfusion injury. This study provides a new therapeutic target for acute ischemia-reperfusion injury and enriches our understanding of the clinical application of CQ10.

\section{Conflicts of Interest}

None of the authors of this paper have any conflicts of interest to declare. 


\section{Authors' Contributions}

Shao Liang and Zhang Ping wrote the manuscript and conducted the research; Jin Ge revised the manuscript and analyzed the dates.

\section{Acknowledgments}

This work was supported by the National Natural Science Foundation of China under Grant no. 81300115 to Shao Liang and Science and Technology for Public Service Project Grants from Wenzhou Municipal Science and the Technology Bureau (Grants Y20150191 to Shao Liang and Y20150114 to Zhang Ping).

\section{References}

[1] N. C. Sacks, A. S. Ash, K. Ghosh, A. K. Rosen, J. B. Wong, and A. B. Rosen, "Trends in acute myocardial infarction hospitalizations: are we seeing the whole picture?," American Heart Journal, vol. 170, no. 6, pp. 1211-1219, 2015.

[2] K. L. Lee, L. H. Woodlief, E. J. Topol et al., "Predictors of 30day mortality in the era of reperfusion for acute myocardial infarction: results from an international trial of 41,021 patients," Circulation, vol. 91, no. 6, pp. 1659-1668, 1995.

[3] J. Herrmann, "Peri-procedural myocardial injury: 2005 update," European Heart Journal, vol. 26, no. 23, pp. 24932519, 2005.

[4] A. Erkol, V. Oduncu, B. Turan et al., "Neutrophil to lymphocyte ratio in acute ST-segment elevation myocardial infarction," The American Journal of the Medical Sciences, vol. 348, no. 1, pp. 37-42, 2014.

[5] J. Kwon, Y. S. Kim, A. S. Cho et al., "The novel role of mast cells in the microenvironment of acute myocardial infarction," Journal of Molecular and Cellular Cardiology, vol. 50, no. 5, pp. 814-825, 2011.

[6] J. J. Sheu, P. H. Sung, S. Leu et al., "Innate immune response after acute myocardial infarction and pharmacomodulatory action of tacrolimus in reducing infarct size and preserving myocardial integrity," Journal of Biomedical Science, vol. 20, no. 1, pp. 82-86, 2013.

[7] L. Song, H. Yang, H. X. Wang et al., "Inhibition of 12/15 lipoxygenase by baicalein reduces myocardial ischemia/reperfusion injury via modulation of multiple signaling pathways," Apoptosis, vol. 19, no. 4, pp. 567-580, 2014.

[8] Y. S. Ang and D. Srivastava, "Oxygen: double-edged sword in cardiac function and repair," Circulation Research, vol. 115, no. 10 , pp. 824-825, 2014.

[9] R. Scherz-Shouval, "Regulation of autophagy by ROS: physiology and pathology," Trends in Biochemical Sciences, vol. 36, no. 1, pp. 30-38, 2011.

[10] N. Mizushima, B. Levine, A. M. Cuervo, and D. J. Klionsky, "Autophagy fights disease through cellular self-digestion," Nature, vol. 451, pp. 1069-1075, 2008.

[11] N. Mizushima and M. Komatsu, "Autophagy: renovation of cells and tissues," Cell, vol. 147, no. 4, pp. 728-741, 2011.

[12] M. Rahman, M. Mofarrahi, A. S. Kristof, B. Nkengfac, S. Harel, and S. N. Hussain, "Reactive oxygen species regulation of autophagy in skeletal muscles," Antioxidants \& Redox Signaling, vol. 20, no. 3, pp. 443-459, 2014.
[13] X. Wen, J. Wu, F. Wang, B. Liu, C. Huang, and Y. Wei, "Deconvoluting the role of reactive oxygen species and autophagy in human diseases," Free Radical Biology \& Medicine, vol. 65, pp. 402-410, 2013.

[14] R. Scherz-Shouval, E. Shvets, E. Fass, H. Shorer, L. Gil, and Z. Elazar, "Reactive oxygen species are essential for autophagy and specifically regulate the activity of Atg4," The EMBO Journal, vol. 26, pp. 1749-1760, 2007.

[15] M. Dhanasekaran and J. Ren, “The emerging role of coenzyme Q-10 in aging, neurodegeneration, cardiovascular disease, cancer and diabetes mellitus," Current Neurovascular Research, vol. 2, no. 5, pp. 447-459, 2005.

[16] M. Antonioli, M. Di Rienzo, M. Piacentini, and G. M. Fimia, "Emerging mechanisms in initiating and terminating autophagy," Trends in Biochemical Sciences, vol. 42, no. 1, pp. 28 41, 2017.

[17] N. Katunuma, A. Matsui, Q. T. Le, K. Utsumi, G. Salvesen, and A. Ohashi, "Novel procaspase-3 activating cascade mediated by lysoapoptases and its biological significances in apoptosis," Advances in Enzyme Regulation, vol. 41, pp. 237-250, 2001.

[18] M. Agosto, M. Azrin, K. Singh, A. S. Jaffe, and B. T. Liang, "Serum caspase-3 p17 fragment is elevated in patients with ST-segment elevation myocardial infarction," Journal of the American College of Cardiology, vol. 57, no. 2, pp. 220-221, 2011.

[19] J. S. Messer, "The cellular autophagy/apoptosis checkpoint during inflammation," Cellular and Molecular Life Sciences, vol. 74, no. 7, pp. 1281-1296, 2017.

[20] N. Makhija, C. Sendasgupta, U. Kiran et al., "The role of oral coenzyme Q10 in patients undergoing coronary artery bypass graft surgery," Journal of Cardiothoracic and Vascular Anesthesia, vol. 22, no. 6, pp. 832-839, 2008.

[21] A. A. Abd-El-Fattah, M. M. El-Sawalhi, E. R. Rashed, and M. A. El-Ghazaly, "Possible role of vitamin E, coenzyme Q10 and rutin in protection against cerebral ischemia/reperfusion injury in irradiated rats," International Journal of Radiation Biology, vol. 86, no. 12, pp. 1070-1078, 2010.

[22] M. Kalayci, M. M. Unal, S. Gul et al., "Effect of coenzyme Q10 on ischemia and neuronal damage in an experimental traumatic brain-injury model in rats," BMC Neuroscience, vol. 12, p. 75, 2011.

[23] C. Bergamini, N. Moruzzi, A. Sblendido, G. Lenaz, and R. Fato, "A water soluble $\mathrm{CoQ}_{10}$ formulation improves intracellular distribution and promotes mitochondrial respiration in cultured cells," PLoS One, vol. 7, no. 3, article e33712, 2012.

[24] M. E. Madmani, A. Yusuf Solaiman, K. Tamr Agha et al., "Coenzyme Q10 for heart failure," Cochrane Database of Systematic Reviews, vol. 6, article CD008684, 2014.

[25] N. Naidoo, R. M. van Dam, W. P. Koh et al., "Plasma vitamin E and coenzyme Q10 are not associated with a lower risk of acute myocardial infarction in Singapore Chinese adults," The Journal of Nutrition, vol. 142, no. 6, pp. 1046-1052, 2012.

[26] I. Zeb, N. Ahmadi, K. Nasir et al., "Aged garlic extract and coenzyme Q10 have favorable effect on inflammatory markers and coronary atherosclerosis progression: a randomized clinical trial," Journal of Cardiovascular Disease Research, vol. 3, no. 3, pp. 185-190, 2012.

[27] L. Shao, D. Wu, P. Zhang et al., "The significance of microthrombosis and fgl2 in no-reflow phenomenon of rats with acute myocardial ischemia/reperfusion," Clinical and Applied Thrombosis/Hemostasis, vol. 19, no. 1, pp. 19-28, 2013. 
[28] B. Redfors, Y. Shao, and E. Omerovic, "Myocardial infarct size and area at risk assessment in mice," Experimental \& Clinical Cardiology, vol. 17, no. 4, pp. 268-272, 2012.

[29] M. A. Farhangi, B. Alipour, E. Jafarvand, and M. Khoshbaten, "Oral coenzyme Q10 supplementation in patients with nonalcoholic fatty liver disease: effects on serum vaspin, chemerin, pentraxin 3, insulin resistance and oxidative stress," Archives of Medical Research, vol. 45, no. 7, pp. 589-595, 2014.

[30] D. A. Groneberg, B. Kindermann, M. Althammer et al., "Coenzyme $\mathrm{Q}_{10}$ affects expression of genes involved in cell signalling, metabolism and transport in human CaCo-2 cells," The International Journal of Biochemistry \& Cell Biology, vol. 37, no. 6, pp. 1208-1218, 2005.

[31] G. G. Schiattarella and J. A. Hill, "Therapeutic targeting of autophagy in cardiovascular disease," Journal of Molecular and Cellular Cardiology, vol. 95, pp. 86-93, 2016.

[32] K. Nishida and K. Otsu, "Autophagy during cardiac remodeling," Journal of Molecular and Cellular Cardiology, vol. 95, pp. 11-18, 2016.

[33] R. F. Villa, A. Gorini, F. Ferrari, and S. Hoyer, "Energy metabolism of cerebral mitochondria during aging, ischemia and post-ischemic recovery assessed by functional proteomics of enzymes," Neurochemistry International, vol. 63, pp. 765781, 2013.

[34] S. Khan, F. Salloum, A. Das, L. Xi, G. W. Vetrovec, and R. C. Kukreja, "Rapamycin confers preconditioning-like protection against ischemia-reperfusion injury in isolated mouse heart and cardiomyocytes," Journal of Molecular and Cellular Cardiology, vol. 41, no. 2, pp. 256-264, 2006.

[35] J. M. Norman, G. M. Cohen, and E. T. Bampton, "The in vitro cleavage of the hAtg proteins by cell death proteases," Autophagy, vol. 6, no. 8, pp. 1042-1056, 2010.

[36] U. M. Nazim, J. H. Moon, J. H. Lee et al., "Activation of autophagy flux by metformin downregulates cellular FLICElike inhibitory protein and enhances TRAIL- induced apoptosis," Oncotarget, vol. 7, no. 17, pp. 23468-23481, 2016. 


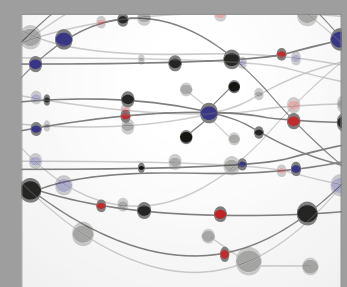

The Scientific World Journal
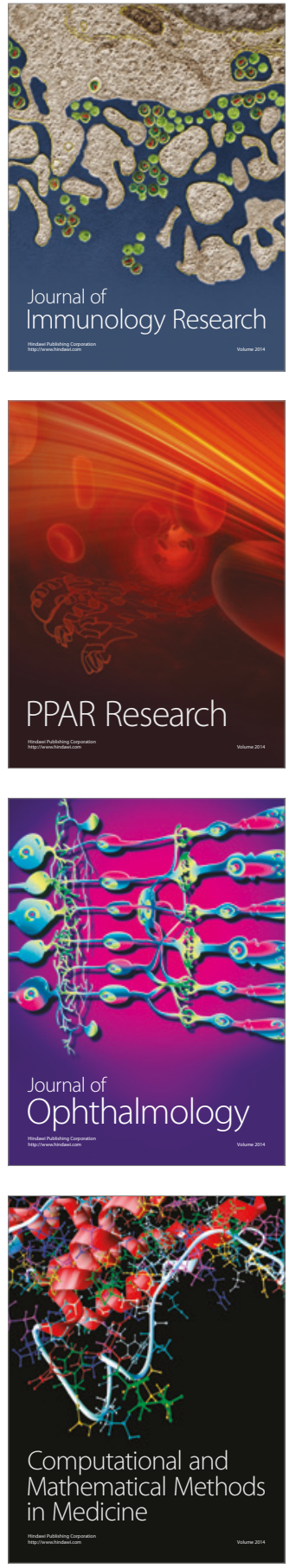

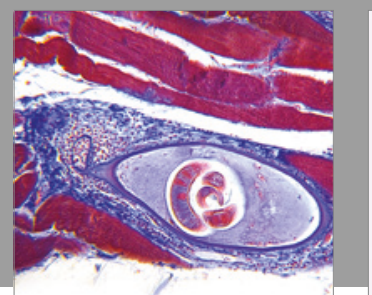

Gastroenterology Research and Practice
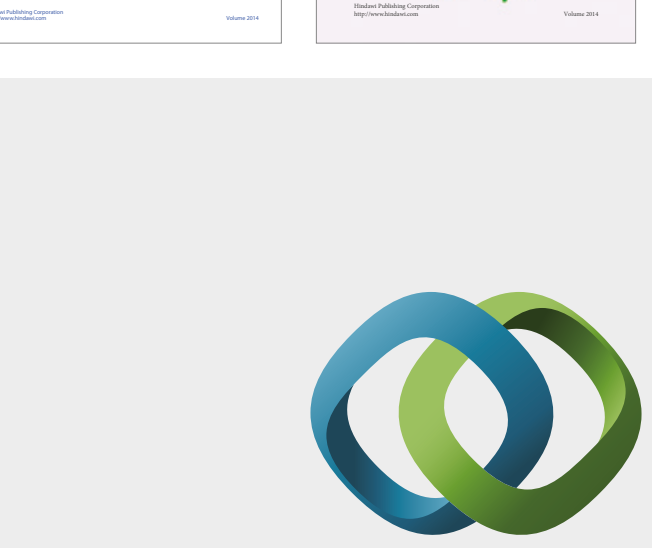

\section{Hindawi}

Submit your manuscripts at

https://www.hindawi.com
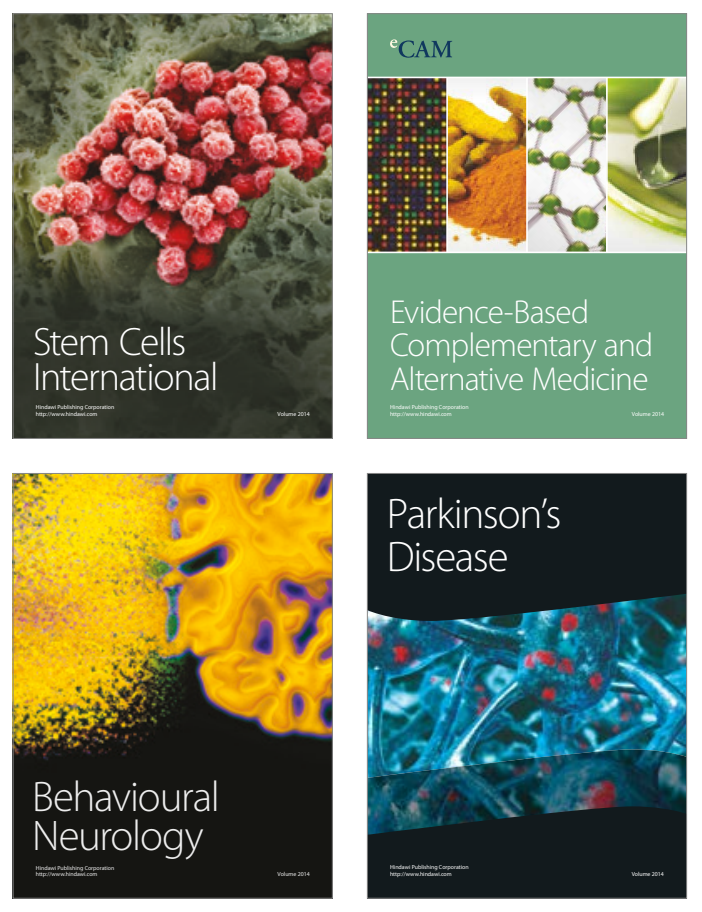
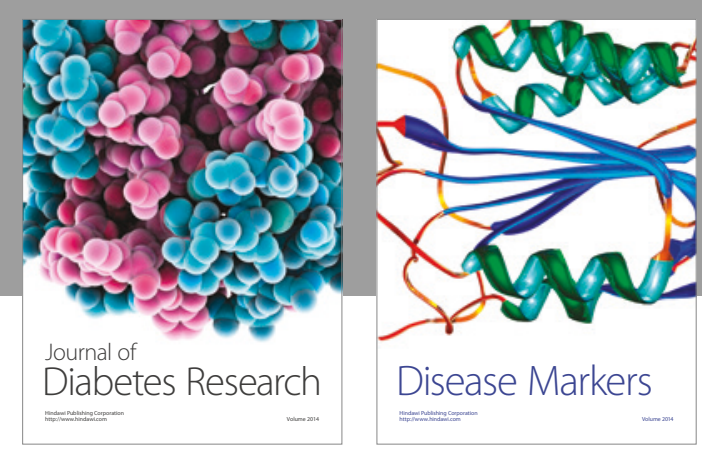

Disease Markers
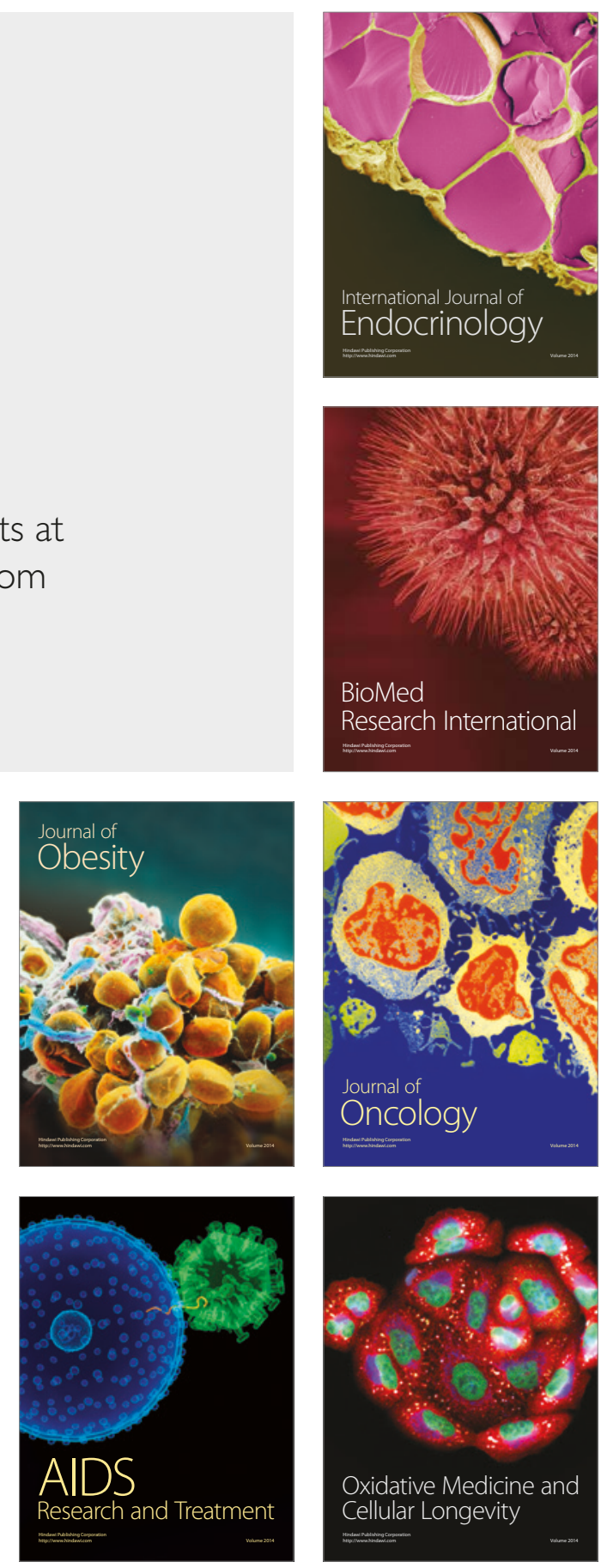